\title{
Quantitative Point of Care testing of HCG in early pregnancy units: A Review
}

\author{
J Bolodeoku1* $^{*}$, A Fakokunde ${ }^{2}$ \\ 1JB Consulting (MDP), 1 Bell Street, Maidenhead, SL6 1BU, United Kingdom \\ 2Department of Obstetrics and Gynaecology, North Middlesex University Hospital, London. N18 1QX, United Kingdom
}

*Corresponding Author: Dr John Bolodeoku, JB Consulting (MDP) Ltd, 1 Bell Street, Maidenhead, SL6 1BU, United Kingdom; Email: John.bolodeoku@jbconsultingmdp. com

Received: May 10, 2019; Accepted: May 24, 2019; Published: June 10, 2019;

\section{Current Practice}

Vaginal bleeding and or abdominal pain occurs in $25 \%-30 \%$ of viable pregnancies and are very common presentations to Primary Care; Emergency Departments and to specialist Early Pregnancy Assessment Units. In up to $42 \%$ of cases, no intrauterine pregnancy is seen on scan (and no ectopic pregnancy is identified) and this is known as a 'pregnancy of unknown location' (PUL) [1]. The current line of investigation for bleeding and pain in early pregnancy is a pelvic ultrasound scan to determine the location and viability of the pregnancy [2]. The pelvic ultrasound scan could be inconclusive in a fair number of patients. In up to $40 \%$ of cases there is no intrauterine pregnancy picked up on the scan, a diagnosis of pregnancy of unknown location (PUL) is considered [3]. Human chorionic gonadotrophin (hCG) is a glycoprotein hormone secreted from the placenta and is the most widely used biomarker as an indication of pregnancy in women. As a single value it is not diagnostic nor beneficial, but when measured serially it is helpful. Serial hCG monitoring and a pelvic ultrasound are the mainstay of management of PUL [4-7]. The expected change in hCG over 48 hours is at least $53 \%$ and gives an indication that the pregnancy (intrauterine or ectopic) is progressing [8]. Serial hCG measurements are therefore used, not to determine the location of the pregnancy, but to predict viability of the pregnancy. The use of serial quantitative human chorionic gonadotropin (hCG) measurements is a mainstay of practice in Early Pregnancy Assessment Units (EPAUs) to aid in the management of these patients.

\section{Quantitative POCT hCG Devices}

The measurement of serial hCG using a recognised laboratory method has been recommended by both the NICE and the Royal College of Obstetrics and Gynaecology guidelines for managing a suspected ectopic pregnancy $[9,10]$. There have been quite a number of qualitative Point of Care (POC) hCG devices available on the market for some time but very few quantitative POC hCG devices. The quantitative POC devices that are currently on the market include the Abbott Point of Care i-STAT, the Radiometer AQT90 FLEX, and the Boditech i-CHROMA ${ }^{\mathrm{TM}}$.

For these devices to become common place in the serial quantification of hCG, there are several questions that need to be answered:
1. What is the accuracy of quantitative POC methods compared to laboratory methods?

2. Are the quantitative POC methods faster when compared to laboratory methods and thereby impact on patient experience (waiting time, decision making, diagnosis and hospital admissions)?

3. What is the cost effectiveness of introducing quantitative POC methods to the treatment pathway?

\section{What is the accuracy of quantitative POC methods compared to laboratory methods?}

The i-STAT is a handheld cartridge-based system, CE certified using whole blood samples, using a sample volume of $17 \mu \mathrm{l}$, with a total assay time of 10 minutes and a working range of $5-2,000 \mathrm{IU} / \mathrm{L}$. Comparative studies between the quantitative POC method i-STAT and existing laboratory methods such as the Abbott Architect Total $\beta$-hCG ; Beckman Dxl Total $\beta$-hCG ; and Roche Cobas e601 hCG $+\beta$ showed that the i-STAT results agreed most closely with the Abbott Architect Total $\beta$-hCG assay, while greater differences were observed with Beckman Dxl Total $\beta$-hCG and Roche Cobas e601 hCG $+\beta$ assays $[11,12]$. (see table 1)

Table 1. Showing correlations $\left(\mathrm{r}^{2}\right)$ between hCG concentrations of i-STAT method and other laboratory methods.

\begin{tabular}{|l|c|}
\hline \multicolumn{1}{|c|}{ Method } & Correlation $\left(\mathbf{r}^{2}\right)$ \\
\hline Beckman Coulter UniCel DX 1800 & $0.994^{11}$ \\
\hline Abbott Architect Total $\beta$-hCG & $0.843^{12}$ \\
\hline Beckman Dxl Total $\beta$-hCG & $0.992^{12}$ \\
\hline Roche Cobas e601 hCG+ $\beta$ & $0.993^{12}$ \\
\hline Abbott Architect Total $\beta$-hCG & $0.993^{12}$ \\
\hline
\end{tabular}

The Boditech i-CHROMA ${ }^{\mathrm{TM}}$ hCG method is a portable device using fluorescence immunoassay (FIA), CE certified using whole blood samples, using a sample volume of $50 \mu \mathrm{l}$, with a total assay time of 15 minutes and a working range of 5-50,000 IU/L. Comparative data between the quantitative POC method Boditech i-CHROMA ${ }^{\mathrm{TM}}$ hCG method and existing laboratory methods such as the Beckman 
Coulter Access2 hCG method described in the product leaflet [13] and in another study [14], with the following methods: Abbott Architect, BioMerieiux VIDAS/mini VIDAS, Roche hCG + Beta, Siemens Centaur XP/XPT/Classic, Siemens Dimension, Siemens DPC Immulite 1000 and 2000, Beckman DxI 600/800, Roche hCG STAT, Beckman Access, SNIBE Maglumi and Ortho Vitros [14] shown in table 2, showed very good correlation. In another study, the Boditech i-CHROMA ${ }^{\mathrm{TM}}$ hCG showed very good correlation with the following methods: Abbott Architect, BioMerieiux VIDAS/mini VIDAS, Roche hCG + Beta, Siemens Centaur XP/XPT/Classic, Siemens Dimension, Siemens DPC Immulite 1000 and 2000, Beckman DxI 600/800, Roche hCG STAT, Beckman Access, SNIBE Maglumi and Ortho Vitros [14] (see table 2).

Table 2. Showing correlations $\left(\mathrm{r}^{2}\right)$ between $\mathrm{hCG}$ concentrations of i-CHROMA ${ }^{\mathrm{TM}}$ method and other laboratory methods

\begin{tabular}{|c|c|}
\hline Method & Correlation $\left(r^{2}\right)$ \\
\hline Beckman Coulter Access ${ }^{2}$ & $0.989^{13}$ \\
\hline Abbott Architect & $0.995^{14}$ \\
\hline Monobind Inc. ELISA/CLIA & $0.842^{14}$ \\
\hline Siemens Centaur CP & $0.992^{14}$ \\
\hline Siemens Centaur XP/XPT/Classic & $0.992^{14}$ \\
\hline Roche Cobas Core EIA & $0.993^{14}$ \\
\hline Beckman DxI 600 /800 & $0.993^{14}$ \\
\hline DiaSorin, Liaison & $0.994^{14}$ \\
\hline Beckman DXI Total $\beta$ hCG (5th IS) & $0.994^{14}$ \\
\hline bioMerieux, VIDAS / mini VIDAS & $0.994^{14}$ \\
\hline Siemens/DPC Immulite 1000 & $0.995^{14}$ \\
\hline SNIBE Maglumi analysers & $0.996^{14}$ \\
\hline Beckman, Access/LXi725 & $0.997^{14}$ \\
\hline Roche $h C G+\beta$ & $0.997^{14}$ \\
\hline Siemens Dimension & 0.99714 \\
\hline Roche hCG STAT (Intact) & $0.998^{14}$ \\
\hline Siemens/DPC Immulite 2000 & $0.998^{14}$ \\
\hline Beckman Access Total $\beta$ hCG (5th IS) & $0.998^{14}$ \\
\hline Ortho Vitros 3600/5600/ECi & $0.998^{14}$ \\
\hline
\end{tabular}

The Radiometer AQT90 method is based on an all in one dry chemistry concept, CE certified using whole blood samples, with a volume $0.3-2 \mathrm{ml}$, with a total assay time of 18 minutes and a working range of $1-5,000 \mathrm{IU} / \mathrm{L}$. The agreement or concordance of the Radiometer AQT90 was 69\% with the Abbott i-STAT, $81 \%$ with the Beckman Coulter and 75\% with the Roche methods [15].
Are the quantitative POC methods faster when compared to laboratory methods and thereby impact on patient experience (waiting time, decision making, diagnosis and hospital admissions)?

The hCG sample, when taken in the emergency unit, is transported to the conventional laboratory and could take approximately 2-3 hours to return. This could affect the patient experience (waiting time, decision making, diagnosis and hospital admissions). A study showed that a quantitative hCG method was simpler and faster than the traditional laboratory method [16]. This is not surprising as the hCG analysis can be done on whole blood samples and the analytical times of these quantitative POC devices range between 10 - 18 minutes, as described in this review. In most cases, in practice, patients are asked to wait until the following day for the result of the hCG test. This not only causes a delay in determining the management plan, it may also result in unnecessary hospital admission and almost certainly increases patient anxiety.

A case scenario and a brief review of the relevant literature was conducted, taking into consideration clinical and analytical elements of the clarity on the use of qualitative and quantitative hCG for the assessment of pregnancy. The conclusion was that use of hCG assays were reliable for pregnancy assessment [17]. A further case was presented from the emergency gynaecology unit at Barts and the London NHS Trust, where a point of care hCG analyser, the Radiometer AQT90 FLEX, was introduced as a strategy to deal with inconclusive sonography results. The unit had previously been sending hCG tests to the laboratory and getting the results back within 2-3 hours. With this POC hCG test, the staff, not being laboratory personnel, found the system reliable; user friendly and very simple to perform the test. In addition, in the past, patients with an inconclusive scan would have been sent away but now they are able to get results with the patients still in the clinic, facilitating the chance to initiate the appropriate treatment for the patient $[18,19]$. More recently, monitoring of serial hCG levels alone, permitted an early viability diagnosis to be made within 48 hours for $41.1 \%$ of patients with PUL, instead of 7 to 14 days with a transvaginal ultrasound scan [20].

\section{The cost effectiveness of introducing quantitative POC devices to the treatment pathway}

There are no studies looking at the cost effectiveness of point of care quantitative hCG testing. However, one study looked at the introduction of a point of care qualitative serum assay for hCG into an outpatient department for a 1 month period and showed a significant decrease in culdocenteses $(\mathrm{p}<0.001)$, ultrasound examinations $(\mathrm{p}<0.025)$ and hospital admissions $(\mathrm{p}<0.01)$, with a net projected institutional reduction in health care costs of $\$ 123,000$ annually [21].

\section{Conclusion}

The possible advantages of a point-of-care quantitative serial hCG test in early pregnancy units would provide a rapid result, helping to aid prompt and effective clinical decision making. It is likely to 
improve patient satisfaction by reducing waiting time for results and clinical decisions and enabling immediate feedback of the results to the patient. It may help diagnoses to be made in primary care, especially in the context of increasing availability of ultrasound scanning in the community setting. In addition, it may allow follow-up in primary care, rather than in secondary care, and it may be more cost effective than current laboratory methods.

In conclusion, there are currently a few POC quantitative hCG testing devices such as the Abbott Point of Care i-STAT, the Radiometer AQT90 FLEX and the Boditech i-CHROMA ${ }^{\mathrm{TM}}$. These devices have demonstrated very good correlation with many laboratory methods. They are all CE approved devices able to measure hCG throughout the acceptable range, using small amounts of whole blood samples assayed all within 18 minutes. The devices should be able to make the patient experience more pleasant by allowing accurate diagnosis to be made, reduce waiting times and hospital admissions and be cost effective.

\section{References}

1. Kirk E, Bottomley C, Bourne T. Diagnosing ectopic pregnancy and current concepts in the management of pregnancy of unknown location. Hum Reprod 2014; 20 (2): 250-261

2. Kirk E, Papageorghiou AT, Condous G, Tan L, Boara S, Bourne T. The diagnostic effectiveness of an initial transvaginal scan in detecting ectopic pregnancy. Hum Reprod 2007; 22 (11): 2824-2828

3. Royal College of Obstetrics and Gynaecologists: The management of tubal ectopic pregnancy. RCOG Guideline No 21. 2010 https://www.rcog.org.uk/en/guidelinesresearch-services/guidelines/gtg21/

4. Banhart KT. Clinical practice. Ectopic pregnancy. N Engl J Med 2009; 361: 379 $-387$

5. Barnhart K, Mennuti MT, Benjamin I, Jacobson S, Goodman D, Coutifaris C Prompt diagnosis of ectopic pregnancy in an emergency department setting. Obstet Gynecol 1994; 84: 1010-1015

6. Nyberg DA, Filly RA, Mahony BS, Monroe S, Laing FC, Jeffrey RB Jr. Early gestation: correlation of HCG levels and sonographic identification. Am J Roentgenol 1985; 144: 951-954

7. Peisner DB, Timor-Tritsch IE. The discriminatory zone of beta-hCG for vaginal probes. J Clin Ultrasound 1990; 18: 280-285

8. Chung K, Allen R.: The use of serial human chorionic gonadotropin levels to establish a viable or a nonviable pregnancy. Semin Reprod Med 2008; 26 (5): 38390 .

9. Royal College of Obstetrics and Gynaecologists. The management of tubal ectopic pregnancy. RCOG Guideline No 21. 210 https://www.rcog.org.uk/en/guidelinesresearch-services/guidelines/gtg21/

10. NICE clinical guideline (CG154). Ectopic pregnancy and miscarriage: Diagnosis and initial management in early pregnancy of ectopic pregnancy and miscarriage. 2012. https://www.nice.org.uk/guidance/cg154

11. Sowder AM, Yarbrough ML, Nerenz RD, Mitsios JV, Mortenson R, Gronowski AM et al. Analytical performance evaluation of the i-STAT total beta-human chorionic gonadotrophin immunoassay. Clin Chim Acta 2015; 446 : $165-170$ Epub 2015/04/29

12. Wikstrom A-K, Hagmar M, Ronquist G, Larsson A. Evaluation of plasma hCG method for point of care testing with the aim of shortening test turnaround times. Open Journal of Obstetrics and Gynecology 2015; 5 : 341-343. http://dx.doi. org/10.4236/ojog.2015.56049

13. $\beta$-hCG Product Leaflet. Boditech Med Inc

14. Bolodeoku J, Bains S, Pinkney S, Coker O, Fakokunde A. Comparison of the Point of Care Test (POCT), i-CHROMA ${ }^{\mathrm{TM}}$ Human Chorionic Gonadotrophin (HCG), Leutinizing Hormone (LH) and Follicle Stimulating Hormone (FSH) methods in serum with the other methods in the Randox International Quality Assessment Scheme (RIQAS). Clin Obstet Gynecol Reprod Med 2017 (4): 1-7

15. Brun MM, Holloway L, Oleksy A, Dayton J, Estey MP, Goudreau B-L, Fuzery AK. Analytical evaluation of the Radiometer AQT90 FLEX 3 hCG assay. Prac Lab Med 2019; 13 :e00116 http://doi.org/10.1016/j.plabm.2019.e00116

16. Von Lode P, Rainaho J, Pettersson K. Quantitative, wide range, 5 minute point of care immunoassay for total human chorionic gonadotrophin in whole blood. Clin Chem 2004; 50 (6): 1026-1035 Epub 2004/04/10
17. Greene DN, Grenache DG. Pathology consultation on human chorionic gonadotropin testing for pregnancy assessment. Am J Clin Pathol 2015; 144 : 830 836. DOI: 10.1309/AJCP707VAREDUYIJ

18. Case Story - Faster point of care hCG testing, transforming patient management in emergency gynaecology unit, reducing patient anxiety. Obs Gynae \& Midwifery News 2012 May 25 http://www.ogpnews.com/2012/05/case-story-faster-point-ofcare-hcg-testing-transforming-patient-management-in-emergency-gynaecologyunit-reducing-patient-anxiety/9581

19. Product News - Point of care hCG invaluable for emergency gynaecology Obs Gynae \& Midwifery News 2011 June 1 http://www.ogpnews.com/2011/06/pointof-care-hcg-testing-invaluable-for-emergency-gynaecology/291

20. Joueidi Y, Bauville E, Laviolle B, Bendavid C, Lavoue V, Le Louis M. Serial hCG and progesterone levels to predict early pregnancy outcomes in pregnancies of uncertain viability: A prospective study. European Journal of Obstetrics and Gynecology and Reproductive Biology 2018; 220: 100-105 https://doi. org/10.1016/j.ejogrb.2017.11.020

21. Gennis P. Gallagher EJ, Andersen F, Hain L. Cost effectiveness of an accurate and rapid assay for serum human chorionic gonadotropin in suspected ectopic pregnancy. Am J of Emerg Med 1988; 6 (1) 4-6

\section{Citation:}

J Bolodeoku, A Fakokunde (2019) Quantitative Point of Care testing of HCG in early pregnancy units: A Review. Integr Gyn Obstet $J$ Volume 2(2): 1-3. 